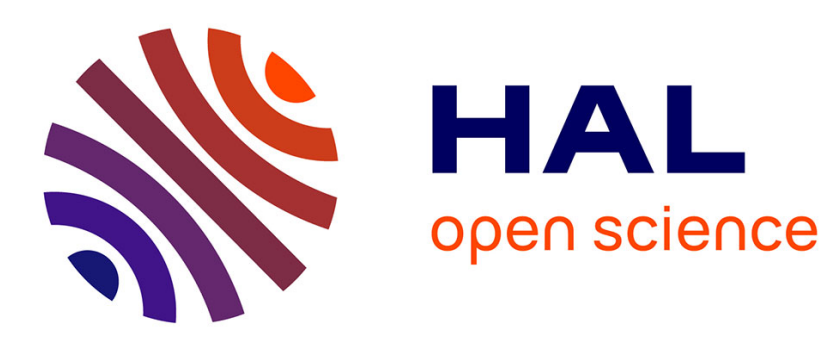

\title{
Light tails: All summands are large when the empirical mean is large
}

Michel Broniatowski, Zhansheng Cao

\section{To cite this version:}

Michel Broniatowski, Zhansheng Cao. Light tails: All summands are large when the empirical mean is large. Extremes, 2014, 17 ( 2), pp.305-336. hal-00813262

\section{HAL Id: hal-00813262 \\ https://hal.science/hal-00813262}

Submitted on 15 Apr 2013

HAL is a multi-disciplinary open access archive for the deposit and dissemination of scientific research documents, whether they are published or not. The documents may come from teaching and research institutions in France or abroad, or from public or private research centers.
L'archive ouverte pluridisciplinaire HAL, est destinée au dépôt et à la diffusion de documents scientifiques de niveau recherche, publiés ou non, émanant des établissements d'enseignement et de recherche français ou étrangers, des laboratoires publics ou privés. 


\title{
Light tails: All summands are large when the empirical mean is large
}

\author{
Michel Broniatowski, Zhansheng Cao \\ LSTA, Université Pierre et Marie Curie, Paris, France
}

\begin{abstract}
It is well known that for a fixed number of independent identically distributed summands with light tail, large values of the sample mean are obtained only when all the summands take large values. This paper explores this property as the number of summands tends to infinity. It provides the order of magnitude of the sample mean for which all summands are in some interval containing this value and it also explores the width of this interval with respect to the distribution of the summands in their upper tail. These results are proved for summands with log-concave or nearly $\log$ concave densities. Making use of some extension of the Erdös-Rényi law of large numbers it explores the forming of aggregates in a sequence of i.i.d. random variables. As a by product the connection is established between large exceedances of the local slope of a random walk on growing bins and the theory of extreme order statistics.

Keywords: random walk;extreme deviation;light tails;conditional limit theorem;Erdös-Rényi law; aggregate forming; extreme values
\end{abstract}

\section{Introduction}

Consider two independent random variables (r.v.'s) with common standard normal distribution on $\mathbb{R}$. The r.v.'s $U:=\left(X_{1}+X_{2}\right) / 2$ and $V:=\left(X_{1}-X_{2}\right) / 2$ are independent, centered and normally distributed. Denote $S_{1}^{2}:=X_{1}+X_{2}$. Then the distribution of $\left(X_{1} / a, X_{2} / a\right)$ given $S_{1}^{2} \geq 2 a$ is that of $(U / a, U / a)+(V / a,-V / a)$ given $U \geq a$. By independence of $U$ and $V$ the pair $(V / a,-V / a)$ goes to $(0,0)$ as $a$ goes to infinity. Since $U$ is gaussian, $U / a$ converges to 1 as $a$ tends to infinity. Thus, given the event $U \geq a$ the pair $\left(X_{1} / a, X_{2} / a\right)$ converges to $(1,1)$ as $a$ tends to infinity. 
This example shows that for fixed $n$, under i.i.d. sampling and in similar cases (in fact independent and with smooth light tail summands), the conditional distribution of $X_{1}^{n}:=\left(X_{1}, . ., X_{n}\right)$ given $\left(S_{1}^{n}:=X_{1}+. .+X_{n} \geq n a\right)$ concentrates on $(a, . ., a)$, the point in $\mathbb{R}^{n}$ with all coordinates equal to $a$ as $a$ tends to infinity. This fact has been considered in [1] and is closely connected with the well known typology of distributions in sub and over exponential distributions. The extension of the above remark from fixed sample size asymptotics to extreme deviations is the starting point of this work.

The question to be addressed can be written as follows:

We assume that the generic random variable is non negative and has a light unbounded tail, namely that its moment generating function is finite in a non void neighborhood of 0 and inf $\left\{x: P\left(X_{1}>x\right)=0\right\}=$ $+\infty$. We consider the statistics $S_{1}^{n}$ defined above.

Assume that for any fixed $n$ it holds

$$
\lim _{a \rightarrow \infty} P\left(X_{1}^{n} \in a B_{n} \mid S_{1}^{n} \geq n a\right)=0
$$

for any Borel set $B_{n}$ in $\mathbb{R}^{n}$ such that $(1, . ., 1) \notin B_{n}$.

Fix such a sequence $B_{n}$ and define $a_{n}$ such that for any $s>a_{n}$

$$
\sup _{s>a_{n}} P\left(X_{1}^{n} \in s B_{n} \mid S_{1}^{n} \geq n s\right) \leq 1 / n .
$$

Such $a_{n}$ surely exists, and is of interest. Obviously we could have defined $a_{n}$ through some other upper bound for the above probability, and the sequence $a_{n}$ depends on the sequence of sets $B_{n}$. The qualitative question raised by the existence of such $a_{n}$ 's is the asymptotic behaviour of the whole sample $X_{1}^{n}$ as $n$ increases when conditioning upon very rare events of the form $\left(S_{1}^{n} \geq n a_{n}\right)$. In other words, our question is:

For which classes of distributions and for which order of magnitude of the conditioning barrier $a_{n}$ do we have

$$
\lim _{n \rightarrow \infty} P\left(\cap X_{i} \in\left(a_{n}-\epsilon_{n}, a_{n}+\epsilon_{n}\right) \mid S_{1}^{n} \geq n a_{n}\right)=1 .
$$

Obviously we concentrate on sequences $a_{n}$ such that

$$
\lim _{n \rightarrow \infty} a_{n}=\infty \text {. }
$$

In the above display the sequence $\epsilon_{n}$ is also a part of the debate: Obviously we require

$$
\lim _{n \rightarrow \infty} \frac{\epsilon_{n}}{a_{n}}=0
$$

which provides the localization of the sample but we also might have the stronger statement

$$
\lim _{n \rightarrow \infty} \epsilon_{n}=0 .
$$


In this event we also might be interested on the rate of decay of $\epsilon_{n}$.

The main result of this paper is a characterization of sequences $a_{n}$ and $\varepsilon_{n}$ according to the form of the density of $X_{1}$. By its very nature this result is strongly dependent upon regularity conditions on the upper tail of the density.

Such questions are also of interest in many branches of physics; see [9] which handles the notion of so-called "democratic localization" of a sample, which corresponds to (1) for fixed $n$ and $a \rightarrow \infty$. The definition of the "democratic localization" property is :"the extreme tail behaviour of the sum $X$ of $N$ random variables comes mostly from contributions where the individual variables in the sum are all close to $X / N$ " (see [9] p80); in [9] and [8] the precise phrasing amounts to assume that the common density of the independent summands is of the form $\exp (-f(x))$ where $f$ satisfies $f^{\prime \prime}>0$ together with $x^{2} f^{\prime \prime}(x) \rightarrow \infty$ as $x \rightarrow \infty$, and the density of the sum is of the form $\exp (-f(x / N))$. Developing this property yields to some form of (1), although not stated as such in the just mentioned works. The present paper is an attempt to formalize this notion. In [9] and [8] the authors explore the consequences of (1) in fragmentation processes and in turbulence, among many others.

The application which is presented in Section 5 pertains to the forming of aggregates in a long run of i.i.d. random variables. The extension of the Erdös-Rényi law of large numbers to the small increments of a random walk obtained in [12] indicates that on the long run with size $N$ of a real valued random walk with i.i.d. increments, the maximum of the local slopes evaluated on moving blocks of summands with size $n(N)$ tends to infinity with $N$, and that this occurs with $n(N)$ going also to infinity, obviously with a low increase. On this very block, and under the hypotheses quoted in this paper, all the summands share nearly the same large value.

The connection with the law of large numbers for the extremes of i.i.d. sequences with light tails (see e.g. [6], Section 5.4) is also explored. In the limit case when $n(N)=1$ the aggregate shrinks to the maximum of the $X_{i}$ 's on the run, which is of the same order as the upper quantile of order $1 / N$ of the distribution of $X$. This is indeed a limit case of the result developed in Section 5, which provides a similar statement for the order of magnitude of the value of the $X_{i}$ 's on the aggregate when $n(N) \rightarrow \infty$. The example when $X_{1}$ has a Weibull tail distribution provides a quantitative measure of this fact.

As a sharpening of the results presented in this paper a companion work provides the approximation of the marginal conditional distribution $P\left(X_{1} \in A \mid S_{1}^{n} \geq n a_{n}\right)$ in variation norm; see [4]. Gibbs conditional theorems have been extensively developed for fixed $a_{n}>E\left(X_{1}\right)$, mostly 
in the case when $X_{1}$ has light tails. The case when $X_{1}$ is substituted by $X_{1}^{k}$ with $k=k_{n}$ has also been considered for fixed $a_{n}$; the extension to extreme deviations $a_{n} \rightarrow \infty$ is a challeging problem which may be useful for the numerical evaluation of small probabilities through Importance Sampling.

\section{Notation and hypotheses}

Denote

$$
C_{n}:=\left(S_{1}^{n} / n>a_{n}\right)
$$

and

$$
I_{n}:=\cap_{i=1}^{n}\left(X_{i} \in\left(a_{n}-\epsilon_{n}, a_{n}+\epsilon_{n}\right)\right) .
$$

The real valued random variables $X_{1}, \ldots, X_{n}$ are independent copies of a r.v. $X$ with density $f$ whose support is $\mathbb{R}^{+}$. As seen by the very nature of the problem handled in this paper, this assumption puts no restriction to the results. We write

$$
f(x):=c \exp (-d(x))
$$

for some positive function $d$, and $c$ is some positive normalizing constant. For $\mathbf{x}:=\left(x_{1} . ., x_{n}\right) \in \mathbb{R}^{n}$ define

$$
I_{d}(\mathbf{x}):=\sum_{1 \leq i \leq n} d\left(x_{i}\right)
$$

and for $A$ a Borel set in $\mathbb{R}^{n}$ denote

$$
I_{d}(A)=\inf _{\mathbf{x} \in A} I_{d}(\mathbf{x})
$$

Two cases will be considered: in the first one $d$ is assumed to be a convex function, and in the second case $d$ will be the sum of a convex function and a "smaller" function in such a way that we will also handle non log-concave densities (although not too far from them). Hence we do not consider heavy tailed r.v. $X$.

For positive $r$ define

$$
S(r)=\left\{\mathbf{x}:=\left(x_{1}, . ., x_{n}\right): \sum_{1 \leq i \leq n} d\left(x_{i}\right) \leq r\right\} .
$$

The notation $f^{\leftarrow}$ denotes the asymptotic inverse function of a function $f$ defined on some interval $(X, \infty)$ and which satisfies $\lim _{x \rightarrow \infty} f(x)=\infty$, through $f^{\leftarrow}(u):=\inf \{x>X$ such that $f(x)>u\}$. 


\subsection{Convex functions and rates of growth of the sequence $a_{n}$}

We now present and discuss regularity assumptions pertaining to the function $d$ when convex. In order to unify the forthcoming notation we denote it $g$ in this case.

A number of regularity conditions are imposed on the asymptotic behaviour of $g$, all commonly met with a reasonable degree of generality. We first define the minimal growth of the sequence $a_{n}$ for which our results hold.

Assume that for some $\delta>0$

$$
\lim \inf _{n \rightarrow \infty} \frac{\log g\left(a_{n}\right)}{\log n}>\delta
$$

holds. The value of $\delta$ will be determined in accordane with the properties of the function $g$.

The function $x \rightarrow g(x)$ is twice differentiable; it satisfies

$$
\lim _{x \rightarrow \infty} g(x) / x=\infty
$$

and $\underline{x}>0$ denotes the smallest real number such that $x \rightarrow g(x)$ is increasing on $(\underline{x}, \infty)$.

We now state the asymptotic condition which links the behaviour of $g$ and the rate of growth of $a_{n}$ as defined through (7); this allows to assemble the puzzle leading to (2).

$$
\begin{aligned}
& \lim _{x \rightarrow \infty} \frac{g(x)^{\frac{1}{\delta}} \log g(2 x)}{x g^{\prime \prime}(x)}=0, \\
& \lim _{x \rightarrow \infty} \frac{g\left(x+\frac{1}{g(x)}\right)-g(x)}{\log g(2 x)}=0 .
\end{aligned}
$$

These conditions are met through a tuning of $\delta$.

\section{The log-concave case}

In this section we assume that $X_{1}, . ., X_{n}$ are i.i.d. with common density $f$ on $\mathbb{R}^{+}$

$$
f(x)=c \exp (-g(x)),
$$

where $g(x)$ satisfies all the conditions in Section 2.1, as does the sequence $a_{n}$.

The following Lemma provides the value of the infimum of the exponent function of the density of the i.i.d. random vector $\left(X_{1}, . ., X_{n}\right)$ on the complementary set of $I_{n}$ and under the constraint that the empirical mean exceeds $a_{n}$. 
Lemma 1 Let (3) hold. Let $\epsilon_{n}$ be a positive sequence such that for large enough $n, a_{n}-\epsilon_{n}>\underline{x}$. Then for such $n$,

$$
I_{g}\left(I_{n}^{c} \cap C_{n}\right)=\min \left(F_{g_{1}}\left(a_{n}, \epsilon_{n}\right), F_{g_{2}}\left(a_{n}, \epsilon_{n}\right)\right),
$$

where

$$
F_{g_{1}}\left(a_{n}, \epsilon_{n}\right)=g\left(a_{n}+\epsilon_{n}\right)+(n-1) g\left(a_{n}-\frac{1}{n-1} \epsilon_{n}\right)
$$

and

$$
F_{g_{2}}\left(a_{n}, \epsilon_{n}\right)=g\left(a_{n}-\epsilon_{n}\right)+(n-1) g\left(a_{n}+\frac{1}{n-1} \epsilon_{n}\right) .
$$

Associated with the functions $F_{g_{1}}$ and $F_{g_{2}}$ we define

$$
\begin{aligned}
& H\left(a_{n}, \epsilon_{n}\right):=\min \left(F_{g_{1}}\left(a_{n}, \epsilon_{n}\right), F_{g_{2}}\left(a_{n}, \epsilon_{n}\right)\right)-n g\left(a_{n}\right) \\
& G\left(a_{n}\right):=g\left(a_{n}+\frac{1}{g\left(a_{n}\right)}\right)-g\left(a_{n}\right) .
\end{aligned}
$$

An explicit calculation of the order of magnitude of $\epsilon_{n}$ with respect to $a_{n}$ envolves the approximation of $H\left(a_{n}, \epsilon_{n}\right)$ and $G\left(a_{n}\right)$. The following Lemma provides sufficient conditions for the existence of sequences $a_{n}$ and $\epsilon_{n}$ satisfying (2).

Lemma 2 When all conditions of Section 2.1 hold, then

$$
\begin{aligned}
\lim _{n \rightarrow \infty} \frac{n \log g\left(a_{n}+\epsilon_{n}\right)}{H\left(a_{n}, \epsilon_{n}\right)} & =0, \\
\lim _{n \rightarrow \infty} \frac{n G\left(a_{n}\right)}{H\left(a_{n}, \epsilon_{n}\right)} & =0 .
\end{aligned}
$$

for some sequence $\epsilon_{n}$ which satisfies (4).

Proof. It follows from standard Taylor expansions in $H\left(a_{n}, \epsilon_{n}\right)$; see the Appendix.

We now state the description of the joint behaviour of the summands $X_{i}$ 's given $(C)$.

Theorem 3 When all conditions of Section 2.1 hold, then there exists a sequence $\epsilon_{n}$ with (4) such that (11) and (12) hold. When both conditions (11) and (12) hold then

$$
\lim _{n \rightarrow \infty} P\left(I_{n} \mid C_{n}\right)=1
$$


Example 4 Let $g(x):=x^{\beta}$. For power functions, through Taylor expansion it holds

$$
g\left(a_{n}+\frac{1}{g\left(a_{n}\right)}\right)-g\left(a_{n}\right)=\frac{\beta}{a_{n}}+o\left(\frac{1}{a_{n}}\right)=o\left(\log g\left(a_{n}\right)\right)
$$

hence condition (12) holds as a consequence of (11). If we assume that $\epsilon_{n}=o\left(a_{n}\right)$, by Taylor expansion we obtain

$$
\min \left(F_{g_{1}}\left(a_{n}, \epsilon_{n}\right), F_{g_{2}}\left(a_{n}, \epsilon_{n}\right)\right)=n a_{n}^{\beta}+C_{\beta}^{2} \frac{n}{n-1} a_{n}^{\beta-2} \epsilon_{n}^{2}+o\left(a_{n}^{\beta-2} \epsilon_{n}^{2}\right) .
$$

Condition (11) then becomes

$$
\lim _{n \rightarrow \infty} \frac{n \log a_{n}}{a_{n}^{\beta-2} \epsilon_{n}^{2}}=0
$$

Case 1: $1<\beta \leq 2$.

To make (13) hold, if we take $a_{n}=n^{\alpha}$ with $a_{n}>1 / \alpha$, we need $\epsilon_{n}$ be large enough, specifically,

$$
a_{n}^{1-\frac{\beta}{2}} \sqrt{n \log a_{n}}=o\left(\epsilon_{n}\right)
$$

which shows that $\epsilon_{n} \rightarrow \infty$.

Case 2: $\beta>2$. In this case, if we take $a_{n}=n^{\alpha}$ with $\alpha>1 /(\beta-2)$, then condition (13) holds for arbitrary sequences $\epsilon_{n}$ bounded by below away from 0 . The sequence $\epsilon_{n}$ may also tend to 0 ; indeed with $\epsilon_{n}=$ $O\left(1 / \log a_{n}\right)$, condition (13) holds.

Example 5 Let $g(x):=e^{x}$. Through Taylor expansion

$$
g\left(a_{n}+\frac{1}{g\left(a_{n}\right)}\right)-g\left(a_{n}\right)=1+o\left(\frac{1}{a_{n}}\right)=o\left(\log g\left(a_{n}\right)\right)=o\left(a_{n}\right),
$$

and if $\epsilon_{n} \rightarrow 0$, it holds

$$
\min \left(F_{g_{1}}\left(a_{n}, \epsilon_{n}\right), F_{g_{2}}\left(a_{n}, \epsilon_{n}\right)\right)=n e^{a_{n}}+\frac{1}{2} \frac{n}{n-1} e^{a_{n}} \epsilon_{n}^{2}+o\left(e^{a_{n}} \epsilon_{n}^{2}\right) .
$$

Hence condition (12) follows from condition (11); furthermore condition (11) follows from

$$
\lim _{n \rightarrow \infty} \frac{n a_{n}}{e^{a_{n}} \epsilon_{n}^{2}}=0
$$

if we set $a_{n}:=n^{\alpha}$ where $\alpha>0$ then condition (12) holds, and $\epsilon_{n}$ is rapidly decreasing to 0 ; indeed we may choose $\epsilon_{n}=o\left(\exp \left(-a_{n} / 4\right)\right)$ together with $\epsilon_{n} / \sqrt{n a_{n} e^{-a_{n}}} \rightarrow \infty$. 
Corollary 6 Let $X_{1}, . ., X_{n}$ be independent r.v's with common Weibull density with shape parameter $k$ and scale parameter 1 ,

$$
f(x)= \begin{cases}k x^{k-1} e^{-x^{k}} & \text { when } x>0 \\ 0 & \text { otherwise }\end{cases}
$$

where $k>2$. Let

$$
a_{n}=n^{\alpha},
$$

for some $\alpha>1 /(k-2)$ and let $\epsilon_{n}$ be a positive sequence tending to 0 such that

$$
\lim _{n \rightarrow \infty} \frac{n \log a_{n}}{a_{n}^{k-2} \epsilon_{n}^{2}}=0
$$

Then

$$
\lim _{n \rightarrow \infty} P\left(I_{n} \mid C_{n}\right)=1
$$

The proof of the above corollary follows from standard expansions and inequalities.

\section{Nearly log-concave densities}

In this section, we pay attention to density functions of the form $f(x)=$ $c \exp (-d(x))$ whose exponent functions $d$ are non-convex functions. Namely, the i.i.d random variables $X_{1}, \ldots, X_{n}$ have common density $f$ with

$$
f(x)=c \exp (-(g(x)+q(x)))
$$

assuming that the convex function $g$ and the sequence $a_{n}$ satisfy all conditions of Section 2.1 and $q(x)$ is of smaller order than $\log g(x)$ for large $x$. We inherit of the definitions of the function $F_{g_{1}}, F_{g_{2}}, G$ and $H$ from the above Section 3 .

Theorem 7 Let $X_{1}, \ldots, X_{n}$ be i.i.d. real valued random variables with common density $f(x)=c \exp (-(g(x)+q(x)))$, where $g(x)$ satisfies the conditions stated in Section 2.1, as does the sequence $a_{n}$. Let $M(x)$ be some nonnegative continuous function on $\mathbb{R}^{+}$for which

$$
-M(x) \leq q(x) \leq M(x) \quad \text { for all positive } x
$$

together with

$$
M(x)=O(\log g(x))
$$

as $x \rightarrow \infty$. Then

$$
\lim _{n \rightarrow \infty} P\left(I_{n} \mid C_{n}\right)=1
$$

and $\epsilon_{n}$ satisfies (4). 
Here are some examples of densities which define r.v's $X_{i}$ 's for which the above Theorem 7 applies. These densities appear in a number of questions pertaining to uniformity in large deviation approximations; see [10], Ch 6.

Example 8 Almost Log-concave densities 1: $f$ can be written as

$$
f(x)=c(x) \exp (-g(x)), \quad 0<x<\infty
$$

with $g$ a twice differentiable convex function with

$$
\lim _{x \rightarrow \infty} g(x) / x=\infty
$$

and where for some $x_{0}>0$ and constants $0<c_{1}<c_{2}<\infty$, we have

$$
c_{1}<c(x)<c_{2} \quad \text { for } \quad x_{0}<x<\infty .
$$

Examples of densities which satisfy the above conditions include the Normal, the hyperbolic density, etc.

Example 9 Almost Log-concave densities 2: A wide class of densities for which our results apply is when there exist constants $x_{0}>0$, $\alpha>0$, and $A$ such that

$$
f(x)=A x^{\alpha-1} l(x) \exp (-g(x)) \quad x>x_{0}
$$

where $l(x)$ is slowly varying at infinity, and $g$ is a twice differentiable convex function which satisfies

$$
\lim _{x \rightarrow \infty} g(x) / x=\infty
$$

All density functions in Examples 8 and 9 satisfy the assumptions of the above Theorem 7 . Also the conditions in Theorem 7 about $a_{n}$ and $\epsilon_{n}$ are the same as those in the convex case, so that if $g(x)$ is some power function with index larger than $2, \epsilon_{n}$ can go to 0 more rapidly than $O\left(1 / \log a_{n}\right)$ (see Example 4); If $g(x)$ is of exponential function form, $\epsilon_{n}$ goes to 0 more rapidly than any power of $1 / a_{n}$ (see Example 5 ).

\section{Aggregate forming and extreme value theory}

\subsection{Improved Erdös-Rényi law and the forming of aggregates}

Consider a long run $X_{1}, \ldots, X_{N}$ of i.i.d. r.v's and let $F$ be the c.d.f of $X_{1}$ which is assumed to satisfy the hypotheses in Theorem 7 , including therefore also Theorem 3 . 
Let

$$
J(x):=\sup _{t} t x-\log E\left(\exp \left(t X_{1}\right)\right)
$$

be the Legendre-Fenchel transform of the moment generating function $t \rightarrow \log E\left(\exp \left(t X_{1}\right)\right)$, which is a strictly convex function defined for all $t>0$.

Denote $\gamma(u):=J^{\leftarrow}(u):=x$ such that $J(x)=u$ the asymptotic inverse function of $J$, a well defined function as $x \rightarrow \infty$.

We assume that the function $\gamma$ is asymptotically equivalent to $u \rightarrow$ $(-\log (1-F))^{\leftarrow}(u)$ in the sense that

$$
\lim _{x \rightarrow \infty} \frac{\gamma(-\log (1-F)(x))}{x}=1 .
$$

This condition holds for many distributions; we refer to [12] for examples and discussion; further results ensuring (15) are discussed hereunder. The following result which is stated in [12] is the starting point of this section.

Let $n(N), 1 \leq n(N) \leq N$ be an integer sequence and denote

$$
M(n(N)):=\max _{0 \leq j \leq N-n(N)} S_{j+1}^{j+n(N)}
$$

the maximum of the sums of the $X_{i}$ 's on blocks of size $n(N)$. Set further

$$
c(n(N)):=\frac{\log N}{n(N)}
$$

assuming

$$
\lim _{N \rightarrow \infty} c(n(N))=\infty .
$$

Under (15) the following result holds

$$
\lim _{N \rightarrow \infty} \frac{M(n(N))}{n(N) \gamma(c(n(N)))}=1 \text { a.s. }
$$

which extends the celebrated Erdös-Rényi law of large numbers to the small increments of the random walk defined through the $X_{i}$ 's. The behaviour of the function $\gamma$ for large values of the argument is closely related to the upper quantile function of $X_{1}$ since under (15)

$$
\gamma(\log x)=(1-F)^{\leftarrow}(1 / x)(1+o(1))
$$

as $x \rightarrow \infty$.

Fix some sequence $n(N)$ satisfying (16) and define for any positive $\delta$ close to 0

$$
a_{n(N), \delta}:=(1-\delta) \gamma(c(n(N)))=:(1-\delta) a_{n(N)} .
$$


By $(18), a_{n(N)}=(1-F)^{\leftarrow}(\exp (-c(n(N)))(1+o(1))$ as $N$ tends to infinity. Due to (17) the choice of $n(N)$ makes

$$
\frac{M(n(N))}{n(N)}>a_{n(N), \delta}
$$

hold ultimately with probability 1 meaning that that for large $N$ there exists at least one block of consecutive $X_{i}{ }^{\prime} s$ with length $n(N)$ whose empirical mean exceeds $a_{n(N), \delta}$. Therefore, by Theorem 7 , all the summands in $X_{j+1}, . ., X_{j+n(N)}$ satisfy

$$
\left|\frac{X_{i}}{a_{n(N), \delta}}-1\right| \leq \frac{\epsilon_{n(N), \delta}}{a_{n(N), \delta}}
$$

with probability going to 1 as $n \rightarrow \infty$. Since $\delta$ is arbitrary, the above property holds with $a_{n(N), \delta}$ substituted by $a_{n(N)}$ and $\epsilon_{n(N)}$ defined accordingly. We may choose the sequences $a_{n}$ and $\epsilon_{n}$ satisfy

$$
\lim _{n \rightarrow \infty} \frac{\epsilon_{n(N)}}{a_{n(N)}}=0
$$

together with the hypotheses in Theorems 3 and 7, which yields

$$
\lim _{n \rightarrow \infty} \frac{X_{i}}{a_{n(N)}}=1
$$

in probability for any $i$ between $j+1$ and $j+n(N)$.

This is the phenomenon of aggregate forming in the random walk. We now show that this phenomenon holds under quite general hypotheses through a discussion on the condition (15).

The case when $-\log (1-F)$ is a regularly varying function When the conditions in Section 3 hold then with $g$ a regularly varying function with index $k>1$, using Theorem 4.12.1 in [2] and Theorem 3.1 in [3] it follows that (15) holds. As an example consider the case when $X_{1}$ has a Weibull distribution on $\mathbb{R}^{+}$with scale parameter 1 and shape parameter $k>1$.

Define $n(N)$ and $a_{n(N)}$ through

$$
n(N)=(\log N)^{\frac{1}{1+k / \alpha}}
$$

for some positive $\alpha$ and

$$
a_{n(N)}:=\gamma\left(\frac{\log N}{n(N)}\right)
$$


which entails, using (18)

$$
a_{n(N)}=(\log N)^{\frac{1}{\alpha+k}}(1+o(1))
$$

Following from the above discussion, there exists a set of consecutive r.v's $X_{j+1}, . ., X_{j+n(N)}$ such that any of the $X_{i}$ 's in this collection is of order $a_{n(N)}$, namely

$$
\lim _{N \rightarrow \infty} \frac{X_{i}}{a_{n(N)}}=1 \text { in probability. }
$$

Reciprocally we may fix a growth condition on $a_{n(N)}$ which measures the height of the aggregate and determine its size $n(N)$. Here are three typical cases:

1-Aggregates with high level: Define for $0<\gamma \leq 1$

$$
a_{n(N)}=(\gamma \log N)^{1 / k} .
$$

Then $n(N)$ tends to a constant. The level is of the order of magnitude of the upper quantile of order $N^{-\gamma}$. This result is a limit case for the results in the present paper since $n(N)$ is bounded; instead it holds as a consequence of the results in [1] together with (17).

2-Aggregates with intermediate level: Define

$$
a_{n(N)}=(\log N)^{\frac{1}{\alpha+k}}
$$

for positive $\alpha$. Then

$$
n(N)=(\log N)^{\frac{1}{1+k / \alpha}}(1+o(1)) .
$$

3-Aggregates with low level: Define

$$
a_{n(N)}=(\gamma \log \log N)^{1 / k} .
$$

Then

$$
n(N)=\frac{\log N}{\gamma \log \log N}(1+o(1))
$$

which are long aggregates.

The case when $-\log (1-F)$ is a rapidly varying function We may also consider cases when $-\log (1-F)$ is a function in some subclass of $\mathcal{R}_{\infty}$, the family of all rapidly varying functions. For this sake we introduce some extra hypotheses on the function $d$ in the exponent of the density of $X_{1}$ as defined in (6), which imply (15), and consequently 
(21). The arguments make use of some tauberian type results pertaining to the Laplace transform of $F$ which yield to introduce

$$
m(t):=\frac{d}{d t} \log E\left(\exp \left(t X_{1}\right)\right)
$$

and

$$
s^{2}(t):=\frac{d^{2}}{d t^{2}} \log E\left(\exp \left(t X_{1}\right)\right)
$$

where the moment generating function $E\left(\exp \left(t X_{1}\right)\right)$ is defined for all non negative $t$ in the present context.

Using the notation of Theorem 7 above, we define

$$
h(x):=g^{\prime}(x)+q^{\prime}(x) .
$$

The regularity condition pertaining to $-\log (1-F)$ which entails (15) makes use of the following definition.

A slowly varying function $l$ which satisfies

$$
\lim _{x \rightarrow \infty} l(x)=\infty
$$

belongs to $\widetilde{R_{0}}$ if it can be represented as

$$
l(x)=\exp \left(\int_{1}^{x} \frac{\epsilon(u)}{u} d u\right), \quad x \geq 1,
$$

where $\epsilon(x)$ is twice differentiable , $\epsilon(x) \rightarrow 0$ as $x \rightarrow \infty$ and

$$
\lim _{x \rightarrow \infty} \frac{x \epsilon^{\prime}(x)}{\epsilon(x)}=0, \quad \lim _{x \rightarrow \infty} \frac{x^{2} \epsilon^{\prime \prime}(x)}{\epsilon(x)}=0
$$

and for some $\eta \in(0,1 / 8)$

$$
\liminf _{x \rightarrow \infty} x^{\eta} \epsilon(x)>0
$$

Remark 10 The class $\widetilde{R_{0}}$ was introduced in [11] in the context of extreme deviation results.

Definition 11 The class $R_{\infty}$ : We say that $h \in R_{\infty}$ if $h$ is ultimately increasing and its inverse function $\psi$ defined through

$$
\psi(u):=h^{\leftarrow}(u)
$$

belongs to $\widetilde{R_{0}}$. 
We assume that for some positive constant $\theta$ and for large $x$ it holds

$$
\sup _{|v-x|<\theta x}|q(x)| \leq \frac{1}{x \sqrt{h(x)}} .
$$

Assuming that (30) holds the two following lemmas hold

Lemma 12 When $h$ belongs to $R_{\infty}$ and (30) holds, then

$$
s^{2}(t)=\psi^{\prime}(t)(1+o(1))
$$

as $t$ tends to infinity.

Proof. See [5], Theorem 3.1.

Lemma 13 If $h \in R_{\infty}$ and then the function $t \rightarrow s^{2}(t)$ is self-neglecting, i.e.

$$
\lim _{t \rightarrow \infty} \sup _{u \in K} \frac{s^{2}\left(t+\frac{u}{s(t)}\right)}{s^{2}(t)}=1
$$

where $K$ is any compact set $K$ in $\mathbb{R}$.

Proof. If $h \in R_{\infty}$, then $\psi(t)$ belongs to $\widetilde{R_{0}}$, hence, using (26)

$$
\psi^{\prime}(t)=\frac{\psi(t)}{t} \epsilon(t) .
$$

Using Lemma 12, we obtain

$$
\frac{1}{s t} \sim \frac{1}{t \sqrt{\psi^{\prime}(t)}}=\sqrt{\frac{1}{t \psi(t) \epsilon(t)}} \longrightarrow 0,
$$

where the last step follows from condition (28). Hence for any $u$ in $K$, we get as $t \rightarrow \infty$

$$
\frac{u}{s(t)}=o(t)
$$

Using the slow variation property of $\psi(t)$ we have, noting $s$ for $s(t)$

$$
\begin{aligned}
\frac{s^{2}(t+u / s)}{s^{2}} & \sim \frac{\psi^{\prime}(t+u / s)}{\psi^{\prime}(t)}=\frac{\psi(t+u / s) \epsilon(t+u / s)}{t+u / s} \frac{t}{\psi(t) \epsilon(t)} \\
& \sim \frac{\epsilon(t+u / s)}{\epsilon(t)}=\frac{\epsilon(t)+O\left(\epsilon^{\prime}(t) u / s\right)}{\epsilon(t)} \longrightarrow 1,
\end{aligned}
$$

where we used a Taylor expansion in the second line, and where the last step holds from condition (27). This completes the proof.

We now state a sufficient condition for (15) to hold. 
Theorem 14 If $h \in R_{\infty}$ and if (30) holds, then (15) holds.

Proof. We make use of the Tauberian results Corollary 1 and Theorem 3 in $[7]$ with the corresponding change of notation. By Lemma 13 condition ii(B) in [7] holds. The other conditions are clearly satisfied, implying

$$
\frac{f}{(1-F)}(x) \sim m^{\leftarrow}(x)=J^{\prime}(x) \quad \text { as } \rightarrow \infty
$$

which together with l'Hôpital rule yields

$$
\lim _{x \rightarrow \infty} \frac{J(x)}{-\log (1-F)(x)}=\lim _{x \rightarrow \infty} \frac{J^{\prime}(x)}{(f /(1-F))(x)}=1 .
$$

It follows that $\gamma:=J^{\leftarrow}$ is a slowly varying function, whence

$$
-\log (1-F)(x)=J(x)(1+o(1))
$$

implies

$$
\begin{aligned}
\gamma(-\log (1-F)(x)) & =\gamma(J(x))(1+o(1)) \\
& =x(1+o(1))
\end{aligned}
$$

as sought.

Example 15 A rapidly varying density. Define $f$ through

$$
f(x)=c \exp \left(-e^{x-1}\right), \quad x \geq 0 .
$$

Then $g(x)=e^{x-1}$ and $q(x)=0$ for all non negative $x$. We show that $h \in R_{\infty}$. It holds $\psi(x)=\log x+1$. Since $h(x)$ is increasing, it remains to show that $\psi(x) \in \widetilde{R_{0}}$. When $x \geq 1, \psi(x)$ admits the representation (26) with $\epsilon(x)=1 /(\log x+1)$. Also conditions (27) and (28) are satisfied. Thus $h \in R_{\infty}$.

Turning back to the aggregate in the long run, (21) holds when $-\log (1-F)$ is a rapidly varying function satisfying $h \in R_{\infty}$, since the sequence $\epsilon_{n}$ can be chosen such that $\epsilon_{n} \rightarrow 0$ rapidly; see Example 5 .

\subsection{Law of large numbers for extreme values and properties of aggregates}

In this section we will show that the above choice for the values of $n(N)$ and of $a_{n(N)}$ is not incidental and bears some insight on the relationship between the size and height of aggregates and the behaviour of extreme terms in a long run. 
Consider the case when $n(N)=1$; this case is not covered by the results in the present paper; however by Theorem 1 in [12], (17) holds, with $a_{1}=\gamma(c(1))$, which by (18) is asymptotically equivalent to the $1 / N$ upper quantile of $F$. In this case the moving block satisfying (20) shrinks to the maximum $X_{N, N}$ of the $X_{i}$ 's in the sample $X_{1}, . ., X_{N}$ for which it is well known that

$$
\frac{X_{N, N}}{a_{1}} \rightarrow 1
$$

a.s. under the current hypotheses in this paper. The extension to the case $n(N)=$ cst holds as a consequence of (17) and the results in [1] for distributions with Weibull type tails, with $a_{1}$ substituted by $a_{c s t}$.

The result which is stated in (20) can be phrased in the following Proposition, which extends in a weak sense this classical result of the theory of extreme order statistics to the behaviour of aggregates in the long run.

Proposition 16 Under the hypotheses of Theorems 3 or 7 and when (15) holds, then with $a_{n(N)}$ defined in (19)

$$
\lim _{N \rightarrow \infty} \min _{0 \leq j \leq N-n(N)}\left(\max _{j \leq i \leq j+n(N)-1}\left|\frac{X_{i}}{a_{n(N)}}-1\right|\right)=0 \text { in probability. }
$$

Remark 17 The relative stability property (32) is known to hold in probability whenever

$$
\lim _{x \rightarrow \infty} \frac{1-F(x \lambda)}{1-F(x)}=0
$$

for all $\lambda>1$ (see e.g. [2], Section 8.13.10).

Remark 18 In the case of the Weibull distribution with shape parameter $k \geq 2$ the sequence $\epsilon_{n}$ can be chosen such that $\lim _{n \rightarrow \infty} \epsilon_{n}=0$ which yields the strengthened form of (33)

$$
\lim _{N \rightarrow \infty} \min _{0 \leq j \leq N-n(N)}\left(\max _{j \leq i \leq j+n(N)-1} X_{i}-a_{n(N)}\right)=0 \text { in probability. }
$$

When $n(N)=1$ this property is known to hold a.s. for all $k>1$, a result which is not obtainable through the results of this paper.

\section{Proofs}

For notational convenience we write $I$ (respectively $C$ ) in place of $I_{n}$ $\left(\right.$ rep. $C_{n}$ ). 


\subsection{Proof of Lemma 1}

Write $\mathbf{x}:=\left(x_{1}, \ldots, x_{n}\right) \in \mathbb{R}_{+}^{n}$, we firstly define the following sets. Let for all $k$ between 0 and $n$

$A_{k}:=\left\{\right.$ there exist $i_{1}, . ., i_{k}$ such that $x_{i_{j}} \geq a_{n}+\epsilon_{n}$ for all $j$ with $\left.1 \leq j \leq k\right\}$

and

$B_{k}:=\left\{\right.$ there exist $i_{1}, . ., i_{k}$ such that $x_{i_{j}} \leq a_{n}-\epsilon_{n}$ for all $j$ with $\left.1 \leq j \leq k\right\}$

In the above definitions it is assumed that the events $A_{k}$ 's are mutually exclusive, and so are the $B_{k}$ 's (i.e. there exist exactly $k$ indexes $i_{1}, . ., i_{k}$ such that the property holds).

Define

$$
A=\bigcup_{k=1}^{n} A_{k}
$$

and

$$
B=\bigcup_{k=1}^{n} B_{k} .
$$

It then holds

$$
I^{c}=A \cup B .
$$

It follows that

$$
\begin{aligned}
I_{g}\left(I^{c} \cap C\right) & =I_{g}((A \cup B) \cap C)=\inf _{\mathbf{x} \in(A \cap C) \cup(B \cap C)} I_{g}(\mathbf{x}) \\
& =\min \left(I_{g}(A \cap C), I_{g}(B \cap C)\right) .
\end{aligned}
$$

Thus we may calculate the minimum values of both $I_{g}(A \cap C)$ and $I_{g}(B \cap C)$ respectively, and finally $I_{g}\left(I^{c} \cap C\right)$.

Step 1: In this step we prove that

$$
I_{g}(A \cap C)=F_{g_{1}}\left(a_{n}, \epsilon_{n}\right) .
$$

Without loss of generality, assume that the $x_{i}$ 's are ordered, $x_{1} \leq \ldots \leq$ $x_{i} \leq x_{i+1} \leq \ldots \leq x_{n}$ and let $i$ and $k:=n-i$ with $1 \leq i \leq n$ such that

$$
x_{1} \leq \ldots \leq x_{i}<a_{n}+\epsilon_{n} \leq x_{i+1} \leq \ldots \leq x_{n}
$$

We first claim that $k<n$. Let $\mathbf{x}_{A \cap C}:=\left(x_{1}, \ldots, x_{n}\right)$ belong to $A \cap C$ and assume that $I_{g}(A \cap C)=I_{g}\left(\mathbf{x}_{A \cap C}\right)$. Indeed let

$$
\mathbf{y}:=\left(y_{1}=a_{n}-\epsilon_{n}, y_{2}=\ldots=y_{n-1}=a_{n}+\epsilon_{n}\right)
$$


which clearly belongs to $A \cap C$. For this $\mathbf{y}$ it holds $I_{g}(\mathbf{y})=(n-1) g\left(a_{n}+\right.$ $\left.\epsilon_{n}\right)+g\left(a_{n}-\epsilon_{n}\right)$ which is strictly smaller than $n g\left(a_{n}+\epsilon_{n}\right)=I_{g}(A \cap C)$ for large $n$. We have proved that $\mathbf{x}_{A \cap C}$ does not belong to $A \cap C$.

Let $\alpha_{i+1}, \ldots, \alpha_{n}$ be nonnegative, and write $x_{i+1}, \ldots, x_{n}$ as

$$
x_{i+1}=a_{n}+\epsilon_{n}+\alpha_{i+1}, \ldots, x_{n}=a_{n}+\epsilon_{n}+\alpha_{n} .
$$

Under condition $(C)$, it holds

$$
\begin{aligned}
x_{1}+\ldots+x_{i} & \geq n a_{n}-\left(x_{i+1}+\ldots+x_{n}\right) \\
& =n a_{n}-k\left(a_{n}+\epsilon_{n}\right)-\left(\alpha_{i+1}+\ldots+\alpha_{n}\right) .
\end{aligned}
$$

Applying Jensen's inequality to the convex function $g$, we have

$$
\begin{aligned}
\sum_{l=1}^{n} g\left(x_{l}\right) & =\left(g\left(x_{i+1}\right)+\ldots+g\left(x_{n}\right)\right)+\left(g\left(x_{1}\right)+\ldots+g\left(x_{i}\right)\right) \\
& \geq\left(g\left(x_{i+1}\right)+\ldots+g\left(x_{n}\right)\right)+(n-k) g\left(x^{*}\right),
\end{aligned}
$$

where equality holds when $x_{1}=\ldots=x_{i}=x^{*}$, with

$$
x^{*}=\frac{n a_{n}-k\left(a_{n}+\epsilon_{n}\right)-\left(\alpha_{i+1}+\ldots+\alpha_{n}\right)}{n-k} .
$$

Define now the function $\left(\alpha_{i+1}, \ldots, \alpha_{n}, k\right) \rightarrow f\left(\alpha_{i+1}, \ldots, \alpha_{n}, k\right)$ through

$$
\begin{aligned}
f\left(\alpha_{i+1}, \ldots, \alpha_{n}, k\right) & =g\left(x_{i+1}\right)+\ldots+g\left(x_{n}\right)+(n-k) g\left(x^{*}\right) \\
& =g\left(a_{n}+\epsilon_{n}+\alpha_{i+1}\right)+\ldots+g\left(a_{n}+\epsilon_{n}+\alpha_{n}\right)+(n-k) g\left(x^{*}\right) .
\end{aligned}
$$

Then $I_{g}(A \cap C)$ is given by

$$
I_{g}(A \cap C)=\inf _{\alpha_{i+1}, \ldots, \alpha_{n} \geq 0,1 \leq k<n} f\left(\alpha_{i+1}, \ldots, \alpha_{n}, k\right) .
$$

We now obtain (34) through the properties of the function $f$. Using (35), the first order partial derivative of $f\left(\alpha_{i+1}, \ldots, \alpha_{n}, k\right)$ with respect to $\alpha_{i+1}$ is

$$
\frac{\partial f\left(\alpha_{i+1}, \ldots, \alpha_{n}, k\right)}{\partial \alpha_{i+1}}=g^{\prime}\left(a_{n}+\epsilon_{n}+\alpha_{i+1}\right)-g^{\prime}\left(x^{*}\right)>0,
$$

where the inequality holds since $g(x)$ is strictly convex and $a_{n}+\epsilon_{n}+$ $\alpha_{i+1}>x^{*}$. Hence $f\left(\alpha_{i+1}, \ldots, \alpha_{n}, k\right)$ is an increasing function with respect to $\alpha_{i+1}$. This implies that the minimum value of $f$ is attained when $\alpha_{i+1}=0$. In the same way, we have $\alpha_{i+1}=\ldots=\alpha_{n}=0$. Therefore it holds

$$
I_{g}(A \cap C)=\inf _{1 \leq k<n} f(\mathbf{0}, k),
$$


with

$$
f(\mathbf{0}, k)=k g\left(a_{n}+\epsilon_{n}\right)+(n-k) g\left(x_{0}^{*}\right),
$$

where

$$
x_{0}^{*}=a_{n}-\frac{k}{n-k} \epsilon_{n} .
$$

Since $f(\mathbf{0}, k)$ is increasing with respect to $k$ its minimal value attains with $k=1$. Thus we have

$$
I_{g}(A \cap C)=f(\mathbf{0}, 1)=F_{g_{1}}\left(a_{n}, \epsilon_{n}\right)
$$

which proves (34).

Step 2: In this step, we follow the same proof as above and prove that

$$
I_{g}(B \cap C)=F_{g_{2}}\left(a_{n}, \epsilon_{n}\right) .
$$

Assume that the $x_{i}$ 's are ranked in ascending order, with $k$ such that $1 \leq k \leq n$ and

$$
x_{1} \leq \ldots \leq x_{k} \leq a_{n}-\epsilon_{n}<x_{k+1} \leq \ldots \leq x_{n}
$$

we obtain $k<n$, otherwise condition $(C)$ won't be satisfied. Denote $x_{1}, \ldots, x_{k}$ by

$$
x_{1}=a_{n}-\epsilon_{n}-\alpha_{1}, \ldots, x_{k}=a_{n}-\epsilon_{n}-\alpha_{k},
$$

where $\alpha_{1}, \ldots, \alpha_{k}$ are nonnegative. Under condition $(C)$, it holds

$$
\begin{aligned}
x_{k+1}+\ldots+x_{n} & \geq n a_{n}-\left(x_{1}+\ldots+x_{k}\right) \\
& =n a_{n}-k\left(a_{n}-\epsilon_{n}\right)+\left(\alpha_{1}+\ldots+\alpha_{k}\right) .
\end{aligned}
$$

Using Jensen's inequality to the convex function $g(x)$, we have

$$
\begin{aligned}
\sum_{l=1}^{n} g\left(x_{l}\right) & =\left(g\left(x_{1}\right)+\ldots+g\left(x_{k}\right)\right)+\left(g\left(x_{k+1}\right)+\ldots+g\left(x_{n}\right)\right) \\
& \geq\left(g\left(x_{1}\right)+\ldots+g\left(x_{k}\right)\right)+(n-k) g\left(x^{\sharp}\right),
\end{aligned}
$$

where the equality holds when $x_{k+1}=\ldots=x_{n}=x^{\sharp}$, with

$$
x^{\sharp}=\frac{n a_{n}-k\left(a_{n}-\epsilon_{n}\right)+\left(\alpha_{1}+\ldots+\alpha_{k}\right)}{n-k} .
$$

Define the function $\left(\alpha_{1}, \ldots, \alpha_{k}, k\right) \rightarrow f\left(\alpha_{1}, \ldots, \alpha_{k}, k\right)$ through

$$
\begin{aligned}
f\left(\alpha_{1}, \ldots, \alpha_{k}, k\right) & =g\left(x_{1}\right)+\ldots+g\left(x_{k}\right)+(n-k) g\left(x^{\sharp}\right) \\
& =g\left(a_{n}-\epsilon_{n}-\alpha_{1}\right)+\ldots+g\left(a_{n}-\epsilon_{n}-\alpha_{k}\right)+(n-k) g\left(x^{\sharp}\right),
\end{aligned}
$$


then $I_{g}(B \cap C)$ is given by

$$
I_{g}(B \cap C)=\inf _{\alpha_{1}, \ldots, \alpha_{k} \geq 0,1 \leq k<n} f\left(\alpha_{1}, \ldots, \alpha_{k}, k\right) .
$$

Using (37), the first order partial derivative of $f\left(\alpha_{1}, \ldots, \alpha_{k}, k\right)$ with respect to $\alpha_{1}$ is

$$
\frac{\partial f\left(\alpha_{1}, \ldots, \alpha_{k}, k\right)}{\partial \alpha_{1}}=-g^{\prime}\left(a_{n}-\epsilon_{n}-\alpha_{1}\right)+g^{\prime}\left(x^{\sharp}\right)>0,
$$

where the inequality holds since $g(x)$ is convex and $a_{n}-\epsilon_{n}-\alpha_{1}<x^{\sharp}$. Hence $f\left(\alpha_{1}, \ldots, \alpha_{k}, k\right)$ is increasing with respect to $\alpha_{1}$. This yields

$$
\alpha_{1}=\ldots=\alpha_{k}=0 \text {. }
$$

Therefore it holds

$$
I_{g}(B \cap C)=\inf _{1 \leq k<n} f(\mathbf{0}, k),
$$

with

$$
f(\mathbf{0}, k)=k g\left(a_{n}-\epsilon_{n}\right)+(n-k) g\left(x_{0}^{\sharp}\right),
$$

where

$$
x_{0}^{\sharp}=a_{n}+\frac{k}{n-k} \epsilon_{n} .
$$

Since the function $y \rightarrow f(\mathbf{0}, y)$ with $0<y<n$ is increasing with respect to $y$, we have

$$
I_{g}(B \cap C)=f(\mathbf{0}, 1)=F_{g_{2}}\left(a_{n}, \epsilon_{n}\right)
$$

which proves the claim.

Thus the proof is completed using (34) and (36).

\subsection{Proof of Lemma 2}

Perform the Taylor expansions

$$
\begin{aligned}
F_{g_{1}}\left(a_{n}, \epsilon_{n}\right)-n g\left(a_{n}\right) & =g\left(a_{n}+\epsilon_{n}\right)+(n-1) g\left(a_{n}-\frac{1}{n-1} \epsilon_{n}\right)-n g\left(a_{n}\right) \\
& =g\left(a_{n}+\epsilon_{n}\right)-g\left(a_{n}\right)-g^{\prime}\left(a_{n}-\theta_{1} \frac{\epsilon_{n}}{n-1}\right) \epsilon_{n} \\
& \geq g\left(a_{n}+\epsilon_{n}\right)-g\left(a_{n}\right)-g^{\prime}\left(a_{n}\right) \epsilon_{n} \\
& =g^{\prime}\left(a_{n}+\theta_{2} \epsilon_{n}\right) \epsilon_{n}-g^{\prime}\left(a_{n}\right) \epsilon_{n}=\theta_{2} g^{\prime \prime}\left(a_{n}+\theta_{3} \theta_{2} \epsilon_{n}\right) \epsilon_{n}^{2},
\end{aligned}
$$

where $\theta_{1}, \theta_{2}, \theta_{3} \in(0,1)$. Hence it holds

$$
\begin{aligned}
\frac{n \log g\left(a_{n}+\epsilon_{n}\right)}{F_{g_{1}}\left(a_{n}, \epsilon_{n}\right)} & \leq \frac{n \log g\left(a_{n}+\epsilon_{n}\right)}{\theta_{2} g^{\prime \prime}\left(a_{n}+\theta_{3} \theta_{2} \epsilon_{n}\right) \epsilon_{n}^{2}} \leq \frac{n \log g\left(2\left(a_{n}+\theta_{3} \theta_{2} \epsilon_{n}\right)\right)}{\theta_{2} g^{\prime \prime}\left(a_{n}+\theta_{3} \theta_{2} \epsilon_{n}\right) \epsilon_{n}^{2}} \\
& \leq \frac{g\left(a_{n}+\theta_{3} \theta_{2} \epsilon_{n}\right)^{\frac{1}{\delta}} \log g\left(2\left(a_{n}+\theta_{3} \theta_{2} \epsilon_{n}\right)\right)}{\theta_{2} g^{\prime \prime}\left(a_{n}+\theta_{3} \theta_{2} \epsilon_{n}\right) \epsilon_{n}^{2}}
\end{aligned}
$$


Under condition (9), there exists $\epsilon_{n}=o\left(a_{n}\right)$ such that the last term of the above display goes to 0 as $a_{n} \rightarrow \infty$. Performing a similar expansion for $F_{g_{2}}\left(a_{n}, \epsilon_{n}\right)-n g\left(a_{n}\right)$, we have shown that (11) holds for some sequence $\epsilon_{n}=o\left(a_{n}\right)$, which satisfies (12) under (10).

\subsection{Proof of Theorem 3}

For $\mathbf{x}:=\left(x_{1}, \ldots, x_{n}\right) \in \mathbb{R}_{+}^{n}$, define

$$
S_{g}(r)=\left\{\mathbf{x}: \sum_{1 \leq i \leq n} g\left(x_{i}\right) \leq r\right\} .
$$

Then for any Borel set $A$ in $\mathbb{R}_{+}^{n}$ it holds

$$
\begin{aligned}
P(A)= & c^{n} \int_{A} \exp \left(-\sum_{1 \leq i \leq n} g\left(x_{i}\right)\right) d x_{1} \ldots d x_{n} \\
& =c^{n} \exp \left(-I_{g}(A)\right) \int_{A} d x_{1} \ldots d x_{n} \int 1_{\left[\sum_{1 \leq i \leq n} g\left(x_{i}\right)-I_{g}(A), \infty\right)}(s) e^{-s} d s \\
& =c^{n} \exp \left(-I_{g}(A)\right) \int_{0}^{\infty} \operatorname{Volume}\left(A \cap S_{g}\left(I_{g}(A)+s\right)\right) e^{-s} d s
\end{aligned}
$$

The proof is divided in three steps; the two first ones explore the numerator and the denominator of

$$
P\left(I^{c} \mid C\right)=\frac{P\left(I^{c} \cap C\right)}{P(C)} .
$$

Step 1: We prove that

$$
P(C) \geq c^{n} \exp \left(-I_{g}(C)-\tau_{n}-n \log g\left(a_{n}\right)\right) .
$$

where

$$
\tau_{n}=n g\left(a_{n}+\frac{1}{g\left(a_{n}\right)}\right)-n g\left(a_{n}\right) .
$$

By convexity of the function $g$, and using condition $(C)$, applying Jensen's inequality, with $x_{1}=\ldots=x_{n}=a_{n}$ it holds

$$
I_{g}(C)=n g\left(a_{n}\right) .
$$

We now consider the largest lower bound for

$$
\log \operatorname{Volume}\left(C \cap S_{g}\left(I_{g}(C)+\tau_{n}\right)\right) .
$$

Denote $B=\left\{\mathbf{x}: x_{i} \in\left[a_{n}, a_{n}+\frac{1}{g\left(a_{n}\right)}\right], i=1, \ldots, n\right\}, S_{g}\left(I_{g}(C)+\tau_{n}\right)=$ $\left\{\mathbf{x}: \sum_{i=1}^{n} g\left(x_{i}\right) \leq n g\left(a_{n}\right)+\tau_{n}\right\}$. 
For large $n$ and any $\mathbf{x}$ in $B$, it holds

$$
\sum_{i=1}^{n} g\left(x_{i}\right) \leq \sum_{i=1}^{n} g\left(a_{n}+\frac{1}{g\left(a_{n}\right)}\right)=n g\left(a_{n}+\frac{1}{g\left(a_{n}\right)}\right)=n g\left(a_{n}\right)+\tau_{n},
$$

where we used the fact that $g(x)$ is an increasing function for large $x$.

Hence

$$
B \subset S_{g}\left(I_{g}(C)+\tau_{n}\right)
$$

It follows that

$\log V$ olume $\left(C \cap S_{g}\left(I_{g}(C)+\tau_{n}\right)\right) \geq \log \operatorname{Volume}(B)=\log \left(\frac{1}{g\left(a_{n}\right)}\right)^{n}=-n \log g\left(a_{n}\right)$

which in turn using (38) and (41a) implies

$$
\begin{aligned}
\log P(C):= & \log c^{n} \int_{C} \exp \left(-\sum_{1 \leq i \leq n} g\left(x_{i}\right)\right) d x_{1} \ldots d x_{n} \\
& \geq n \log c+\log \left(\exp \left(-I_{g}(C)\right) \int_{\tau_{n}}^{\infty} \operatorname{Volume}\left(C \cap S_{g}\left(I_{g}(C)+s\right)\right) e^{-s} d s\right) \\
& \geq n \log c-I_{g}(C)-\tau_{n}+\log \operatorname{Volume}\left(C \cap S_{g}\left(I_{g}(C)+\tau_{n}\right)\right) \\
& \geq n \log c-I_{g}(C)-\tau_{n}-n \log g\left(a_{n}\right),
\end{aligned}
$$

This proves the claim.

Step 2: In this step, we prove that

$$
P\left(I^{c} \cap C\right) \leq c^{n} \exp \left(-I_{g}\left(I^{c} \cap C\right)+n \log I_{g}\left(I^{c} \cap C\right)+\log (n+1)\right) .
$$

Denote $A_{n}:=I^{c} \cap C$. For positive $s$, let

$$
S_{g}\left(I_{g}\left(A_{n}\right)+s\right)=\left\{\mathbf{x}: \sum_{1 \leq i \leq n} g\left(x_{i}\right) \leq I_{g}\left(A_{n}\right)+s\right\}
$$

and

$$
F=\left\{\mathbf{x}: g\left(x_{i}\right) \leq I_{g}\left(A_{n}\right)+s, i=1, \ldots, n\right\}
$$

It holds

$$
S_{g}\left(I_{g}\left(A_{n}\right)+s\right) \subset F .
$$

Since $\lim _{x \rightarrow \infty} g(x) / x=+\infty$,

$$
F \subset\left\{\mathbf{x}: x_{i} \leq\left(I_{g}\left(A_{n}\right)+s\right), i=1, \ldots, n\right\}
$$

which yields

$$
S_{g}\left(I_{g}\left(A_{n}\right)+s\right) \subset\left\{\mathbf{x}: x_{i} \leq\left(I_{g}\left(A_{n}\right)+s\right), i=1, \ldots, n\right\},
$$


from which we obtain

$\operatorname{Volume}\left(A_{n} \cap S_{g}\left(I_{g}\left(A_{n}\right)+s\right)\right) \leq \operatorname{Volume}\left(S_{g}\left(I_{g}\left(A_{n}\right)+s\right)\right) \leq\left(I_{g}\left(A_{n}\right)+s\right)^{n}$.

With this inequality and (38) we get as $a_{n} \rightarrow \infty$

$$
\begin{aligned}
\log P\left(A_{n}\right)= & \log c^{n} \int_{A_{n}} \exp \left(-\sum_{1 \leq i \leq n} g\left(x_{i}\right)\right) d x_{1} \ldots d x_{n} \\
& =n \log c-I_{g}\left(A_{n}\right)+\log \int_{0}^{\infty} \operatorname{Volume}\left(A_{n} \cap S_{g}\left(I_{g}\left(A_{n}\right)+s\right)\right) e^{-s} d s \\
& \leq n \log c-I_{g}\left(A_{n}\right)+\log \int_{0}^{\infty}\left(I_{g}\left(A_{n}\right)+s\right)^{n} e^{-s} d s .
\end{aligned}
$$

It holds

$$
\begin{aligned}
& \int_{0}^{\infty}\left(I_{g}\left(A_{n}\right)+s\right)^{n} e^{-s} d s \\
& =I_{g}\left(A_{n}\right)^{n}+n \int_{0}^{\infty}\left(I_{g}\left(A_{n}\right)+s\right)^{n-1} e^{-s} d s \\
& =I_{g}\left(A_{n}\right)^{n}+n I_{g}\left(A_{n}\right)^{n-1}+n(n-1) \int_{0}^{\infty}\left(I_{g}\left(A_{n}\right)+s\right)^{n-2} e^{-s} d s
\end{aligned}
$$

where the second term in the last display is negligible with respect to the first one, as $I_{g}\left(A_{n}\right) \geq n g\left(a_{n}\right)$ and $a_{n} \rightarrow \infty$. Iterating the integration by parts and using the same argument we obtain the upper bound

$$
\int_{0}^{\infty}\left(I_{g}\left(A_{n}\right)+s\right)^{n} e^{-s} d s \leq(n+1) I_{g}\left(A_{n}\right)^{n} .
$$

Hence we have

$$
\begin{aligned}
\log P\left(A_{n}\right) & \leq n \log c-I_{g}\left(A_{n}\right)+\log \left((n+1) I_{g}\left(A_{n}\right)^{n}\right) \\
& =n \log c-I_{g}\left(A_{n}\right)+n \log I_{g}\left(A_{n}\right)+\log (n+1)
\end{aligned}
$$

as sought.

Step 3: In this step, we will complete the proof, showing that

$$
\lim _{a_{n} \rightarrow \infty} \frac{P\left(I^{c} \cap C\right)}{P(C)}=0 .
$$

By Lemma 1,

$$
I_{g}\left(I^{c} \cap C\right)=\min \left(F_{g_{1}}\left(a_{n}, \epsilon_{n}\right), F_{g_{2}}\left(a_{n}, \epsilon_{n}\right)\right) .
$$


Using (39) and (42) it holds

$\frac{P\left(I^{c} \cap C\right)}{P(C)} \leq \exp \left(-H\left(a_{n}, \epsilon_{n}\right)+n \log I_{g}\left(I^{c} \cap C\right)+\tau_{n}+n \log g\left(a_{n}\right)+\log (n+1)\right)$.

Under conditions (12), by (40) when $a_{n} \rightarrow \infty$, we have

$$
\frac{\tau_{n}}{H\left(a_{n}, \epsilon_{n}\right)}=\frac{n G\left(a_{n}\right)}{H\left(a_{n}, \epsilon_{n}\right)} \longrightarrow 0
$$

Using condition (11), when $a_{n} \rightarrow \infty$,

$$
\frac{n \log g\left(a_{n}\right)}{H\left(a_{n}, \epsilon_{n}\right)} \longrightarrow 0, \quad \text { and } \quad \frac{\log (n+1)}{H\left(a_{n}, \epsilon_{n}\right)} \longrightarrow 0 .
$$

As to the term $n \log I_{g}\left(I^{c} \cap C\right)$, we have

$$
\begin{aligned}
n \log I_{g}\left(I^{c} \cap C\right) & =n \log \min \left(F_{g_{1}}\left(a_{n}, \epsilon_{n}\right), F_{g_{2}}\left(a_{n}, \epsilon_{n}\right)\right) \\
& \leq n \log \left(n g\left(a_{n}+\epsilon_{n}\right)\right) \\
& =n \log n+n \log g\left(a_{n}+\epsilon_{n}\right) .
\end{aligned}
$$

Under condition (11), $n \log g\left(a_{n}+\epsilon_{n}\right)$ is of small order with respect to $H\left(a_{n}, \epsilon_{n}\right)$ as $n$ tends to infinity. Under condition (7), for $a_{n}$ large enough, there exists some positive constant $Q$ such that $\log n \leq Q \log g\left(a_{n}\right)$. Hence we have

$$
n \log n \leq Q n \log g\left(a_{n}\right)
$$

which under condition (11), yields that $n \log n$ is negligible with respect to $H\left(a_{n}, \epsilon_{n}\right)$. Hence when $a_{n} \rightarrow \infty$, it holds

$$
\frac{n \log \left(I_{g}\left(I^{c} \cap C\right)\right)}{H\left(a_{n}, \epsilon_{n}\right)} \longrightarrow 0 .
$$

Further, (46), (47) and (45) make (44) hold. This completes the proof.

\subsection{Proof of Theorem 7}

The proof uses the same argument as in Theorem7, adding some technicalities. A lower (rep. upper) envelope $g_{1}$ (resp. $g_{2}$ ) for the function $g+q$ are introduced.

In Step 1, using a convex envelope for $g+q$, we bound $I_{g, q}(C)$ (defined hereunder in (48)) which is used to bound $P(C)$ from below in Step 2.

Step 3 provides the upper bound of $P\left(I^{c} \cap C\right)$ in terms of $I_{g, q}\left(I^{c} \cap C\right)$ and $\log I_{g}\left(I^{c} \cap C\right)$. 
In Step 4, we obtain crude bounds of $I_{g_{1}}\left(I^{c} \cap C\right), I_{g_{2}}(C)$ and $\log I_{g_{2}}\left(I^{c} \cap\right.$ $C$ ), which are used to provide upper (or lower) bounds, respectively, for $I_{g, q}\left(I^{c} \cap C\right), I_{g, q}(C)$ and $\log I_{g, q}\left(I^{c} \cap C\right)$ in Step 5. The proof is completed with the help of these bounds.

Denote $\mathbf{x}=\left(x_{1}, \ldots, x_{n}\right)$ in $\mathbb{R}_{+}^{n}$ and, for a Borel set $A \in \mathbb{R}_{+}^{n}$ define

$$
I_{g, q}(A)=\inf _{\mathbf{x} \in A} I_{g, q}(\mathbf{x})
$$

where

$$
I_{g, q}(\mathbf{x}):=\sum_{1 \leq i \leq n}\left(g\left(x_{i}\right)+q\left(x_{i}\right)\right) .
$$

Also for any positive $r$ define

$$
S_{g, q}(r)=\left\{\mathbf{x}: \sum_{1 \leq i \leq n}\left(g\left(x_{i}\right)+q\left(x_{i}\right)\right) \leq r\right\} .
$$

Then it holds

$$
\begin{aligned}
& P(A)=c^{n} \int_{A} \exp \left(-\sum_{1 \leq i \leq n}\left(g\left(x_{i}\right)+q\left(x_{i}\right)\right)\right) d x_{1}, \ldots, d x_{n} \\
& =c^{n} \exp \left(-I_{g, q}(A)\right) \int_{A} d x_{1}, \ldots, d x_{n} \int 1_{\left[\sum_{1 \leq i \leq n}\left(g\left(x_{i}\right)+q\left(x_{i}\right)\right)-I_{g, q}(A), \infty\right)}(s) e^{-s} d s \\
& =c^{n} \exp \left(-I_{g, q}(A)\right) \int_{0}^{\infty} \operatorname{Volume}\left(A \cap S_{g, q}\left(I_{g, q}(A)+s\right)\right) e^{-s} d s
\end{aligned}
$$

Step 1: In this step we prove that

$$
I_{g, q}(C) \geq n g\left(a_{n}\right)-n N \log g\left(a_{n}\right)
$$

for some positive constant $N$.

For large $x$ it holds

$$
g(x)-M(x) \leq g(x)+q(x) \leq g(x)+M(x) .
$$

Set $g_{1}(x)=g(x)-M(x)$ and $g_{2}(x)=g(x)+M(x)$, then it follows

$$
I_{g_{1}}(C) \leq I_{g, q}(C) \leq I_{g_{2}}(C) .
$$

In the same way, it holds

$$
I_{g_{1}}\left(I^{c} \cap C\right) \leq I_{g, q}\left(I^{c} \cap C\right) \leq I_{g_{2}}\left(I^{c} \cap C\right) .
$$


By condition (14), there exists some large positive $y_{0}$ and some positive constant $N$ such that for $x \in\left[y_{0}, \infty\right)$

$$
M(x) \leq N \log g(x) .
$$

Set $r(x)=g(x)-N \log g(x)$; the second order derivative of $r(x)$ is

$$
r^{\prime \prime}(x)=g^{\prime \prime}(x)\left(1-\frac{N}{g(x)}\right)+\frac{N\left(g^{\prime}(x)\right)^{2}}{g^{2}(x)},
$$

where the second term is positive. The function $g$ is increasing on some interval $[X, \infty)$ where we also have $g(x)>x$. Hence there exists some $y_{1} \in[X, \infty)$ such that $g(x)>N$ when $x \in\left[y_{1}, \infty\right)$. This implies that $r^{\prime \prime}(x)>0$ and $r^{\prime}(x)>0$ and therefore $r(x)$ is convex and increasing on $\left[y_{1}, \infty\right)$.

In addition, $M(x)$ is bounded on any finite interval; there exists some $y_{2} \in\left[y_{1}, \infty\right)$ such that for all $x \in\left(0, y_{2}\right)$

$$
M(x) \leq N \log g\left(y_{2}\right)
$$

The function $g$ is convex and increasing on $\left[y_{2}, \infty\right)$. Thus there exists $y_{3}$ such that

$$
g^{\prime}\left(y_{3}\right)>2 g^{\prime}\left(y_{2}\right) \text { and } g\left(y_{3}\right)>2 N .
$$

We now construct a function $h$ as follows. Let

$$
h(x)=r(x) \mathbf{1}_{\left[y_{3}, \infty\right)}(x)+s(x) \mathbf{1}_{\left(0, y_{3}\right)}(x),
$$

where $s(x)$ is defined by

$$
s(x)=r\left(y_{3}\right)+r^{\prime}\left(y_{3}\right)\left(x-y_{3}\right) .
$$

We will show that

$$
g_{1}(x) \geq h(x)
$$

for $x \in(0, \infty)$.

If $x \in\left[y_{3}, \infty\right)$, then by (53), it holds

$$
h(x)=r(x)=g(x)-N \log g(x) \leq g(x)-M(x)=g_{1}(x) .
$$

If $x \in\left(y_{2}, y_{3}\right)$, using (57), we have

$$
s(x) \leq r(x)=g(x)-N \log g(x) \leq g(x)-M(x)=g_{1}(x),
$$

where the first inequality comes from the convexity of $r(x)$. We now show that (58) holds when $x \in\left(0, y_{2}\right]$ if $y_{3}$ is large enough. For this purpose, set

$$
t(x)=g(x)-s(x)-N \log g\left(y_{2}\right) .
$$


Take the first order derivative of $t$ and use the convexity of $g$ on $\left(0, y_{2}\right]$. We have

$$
\begin{aligned}
t^{\prime}(x) & =g^{\prime}(x)-s^{\prime}(x)=g^{\prime}(x)-r^{\prime}\left(y_{3}\right)=g^{\prime}(x)-\left(g^{\prime}\left(y_{3}\right)-\frac{N g^{\prime}\left(y_{3}\right)}{g\left(y_{3}\right)}\right) \\
& =g^{\prime}(x)-\left(1-\frac{N}{g\left(y_{3}\right)}\right) g^{\prime}\left(y_{3}\right) \leq g^{\prime}\left(y_{2}\right)-\left(1-\frac{N}{g\left(y_{3}\right)}\right) g^{\prime}\left(y_{3}\right) \\
& <\frac{1}{2} g^{\prime}\left(y_{3}\right)-\left(1-\frac{N}{g\left(y_{3}\right)}\right) g^{\prime}\left(y_{3}\right)<0,
\end{aligned}
$$

where the inequalities in the last line hold from (55). Therefore $t$ is decreasing on $\left(0, y_{2}\right]$. It follows that

$t(x) \geq t\left(y_{2}\right)=g\left(y_{2}\right)-N \log g\left(y_{2}\right)-s\left(y_{2}\right) \geq g\left(y_{2}\right)-N \log g\left(y_{2}\right)-r\left(y_{2}\right)=0$,

which, together with (54), yields, when $x \in\left(0, y_{2}\right]$

$$
g_{1}(x)=g(x)-M(x) \geq g(x)-N \log g\left(y_{2}\right) \geq s(x) .
$$

Together with (59), (60) and (56), this last display means that (58) holds.

We now prove that $h$ is a convex function on $(0, \infty)$; indeed for $x$ such that $0<x \leq y_{3}, h^{\prime \prime}(x)=0$, and if $x>y_{3}, h^{\prime \prime}(x)=r^{\prime \prime}(x)>0$. The left derivative of $h(x)$ at $y_{3}$ is $h^{\prime}\left(y_{3}^{-}\right)=r^{\prime}\left(y_{3}\right)$, and it is obvious that the right derivative of $h(x)$ at $y_{3}$ is also $h^{\prime}\left(y_{3}^{+}\right)=r^{\prime}\left(y_{3}\right)$; hence $h$ is derivable at $y_{3}$ and $h^{\prime}\left(y_{3}\right)=r^{\prime}\left(y_{3}\right)$; hence $h^{\prime \prime}\left(y_{3}\right)=r^{\prime \prime}\left(y_{3}\right)>0$. This shows that $h$ is convex on $(0, \infty)$.

Now under condition $(C)$, using the convexity of $h$ and (58), it holds

$$
I_{g_{1}}(\mathbf{x})=\sum_{i=1}^{n}\left(g\left(x_{i}\right)-M\left(x_{i}\right)\right) \geq \sum_{i=1}^{n} h\left(x_{i}\right) \geq n h\left(\frac{\sum_{i=1}^{n} x_{i}}{n}\right)=n h\left(a_{n}\right) .
$$

Using (51), we obtain the lower bound of $I_{g, q}(C)$ under condition $(C)$ for $a_{n}$ large enough (say, $a_{n}>y_{3}$ )

$$
I_{g, q}(C) \geq I_{g_{1}}(C) \geq n h\left(a_{n}\right)=n r\left(a_{n}\right)=n g\left(a_{n}\right)-n N \log g\left(a_{n}\right) .
$$

Step 2: In this step, we will show that the following lower bound of $P(C)$ holds

$$
P(C) \geq c^{n} \exp \left(-I_{g, q}(C)-\tau_{n}-n \log g\left(a_{n}\right)\right),
$$

where $\tau_{n}$ is defined by

$$
\begin{aligned}
\tau_{n} & =n g\left(a_{n}+\frac{1}{g\left(a_{n}\right)}\right)-n g\left(a_{n}\right)+n N \log g\left(a_{n}+\frac{1}{g\left(a_{n}\right)}\right)+n N \log g\left(a_{n}\right) \\
& =n G\left(a_{n}\right)+n N \log g\left(a_{n}\right)+n N \log g\left(a_{n}+\frac{1}{g\left(a_{n}\right)}\right)
\end{aligned}
$$


Denote $B=\left\{\mathbf{x}: x_{i} \in\left[a_{n}, a_{n}+\frac{1}{g\left(a_{n}\right)}\right], i=1, \ldots, n\right\}$. If $\mathbf{x} \in B$, by (53), which holds for large $a_{n}$ (say, $a_{n}>y_{3}$ and $g$ is an increasing function on $\left.\left(y_{3}, \infty\right)\right)$, we have

$$
\begin{aligned}
I_{g, q}(\mathbf{x}) & \leq \sum_{i=1}^{n}\left(g\left(x_{i}\right)+M\left(x_{i}\right)\right) \leq \sum_{i=1}^{n}\left(g\left(x_{i}\right)+N \log g\left(x_{i}\right)\right) \\
& \leq \sum_{i=1}^{n}\left(g\left(a_{n}+\frac{1}{g\left(a_{n}\right)}\right)+N \log g\left(a_{n}+\frac{1}{g\left(a_{n}\right)}\right)\right) \\
& =n g\left(a_{n}+\frac{1}{g\left(a_{n}\right)}\right)+n N \log g\left(a_{n}+\frac{1}{g\left(a_{n}\right)}\right) \\
& =\tau_{n}+n g\left(a_{n}\right)-n N \log g\left(a_{n}\right) \leq \tau_{n}+I_{g, q}(C)
\end{aligned}
$$

where the last inequality holds from (61). Hence

$$
B \subset S_{g, q}\left(I_{g, q}(C)+\tau_{n}\right) .43
$$

Since $B \subset C$, we have

$$
B \subset C \cap S_{g, q}\left(I_{g, q}(C)+\tau_{n}\right) .
$$

Now we may obtain the lower bound

$\log \operatorname{Volume}\left(C \cap S_{g, q}\left(I_{g, q}(C)+\tau_{n}\right)\right) \geq \log \operatorname{Volume}(B)=-n \log g\left(a_{n}\right)$.

Using (49) and (64), it holds

$$
\begin{aligned}
\log P(C) & =\log c^{n} \int_{C} \exp \left(-\sum_{1 \leq i \leq n}\left(g\left(x_{i}\right)+q\left(x_{i}\right)\right)\right) d x_{1}, \ldots, d x_{n} \\
& =n \log c-I_{g, q}(C)+\log \int_{0}^{\infty} \operatorname{Volume}\left(C \cap S_{g, q}\left(I_{g, q}(C)+s\right)\right) e^{-s} d s \\
& \geq n \log c-I_{g, q}(C)+\log \int_{\tau_{n}}^{\infty} \operatorname{Volume}\left(C \cap S_{g, q}\left(I_{g, q}(C)+\tau_{n}\right)\right) e^{-s} d s \\
& \geq n \log c-I_{g, q}(C)-\tau_{n}-n \log g\left(a_{n}\right),
\end{aligned}
$$

so (62) holds.

Step 3: We prove that

$P\left(I^{c} \cap C\right) \leq c^{n} \exp \left(-I_{g, q}\left(I^{c} \cap C\right)+n \log I_{g}\left(I^{c} \cap C\right)+\log (n+1)+n \log 2\right)$.

Similarly as in step 2 of the proof of Theorem 3 denote

$$
A_{n}:=I^{c} \cap C .
$$


For any positive $s$,

$$
S_{g, q}\left(I_{g, q}\left(A_{n}\right)+s\right)=\left\{\mathrm{x}: \sum_{1 \leq i \leq n}\left(g\left(x_{i}\right)+q\left(x_{i}\right)\right) \leq I_{g, q}\left(A_{n}\right)+s\right\}
$$

is included in $\left\{\mathbf{x}: g\left(x_{i}\right)+q\left(x_{i}\right) \leq I_{g, q}\left(A_{n}\right)+s, i=1, \ldots, n\right\}$ which in turn is included in $F=\left\{\mathbf{x}: g\left(x_{i}\right)-M\left(x_{i}\right) \leq\left(I_{g, q}\left(A_{n}\right)+s\right), i=1, \ldots, n\right\}$ by (50).

Set $H=\left\{\mathbf{x}:=\left(x_{1}, . ., x_{n}\right): x_{i} \leq 2\left(I_{g, q}\left(A_{n}\right)+s\right), i=1, \ldots, n\right\}$, we will show that for $a_{n}$ large enough

$$
F \subset H \text {. }
$$

Suppose that for some $\mathbf{x}:=\left(x_{1}, . ., x_{n}\right)$ in $F$, some $x_{i}$ is larger than $2\left(I_{g, q}(\right.$ $\left.\left.A_{n}\right)+s\right)$. For $a_{n}$ large enough, by (61), it holds

$$
\begin{aligned}
x_{i} & \geq 2\left(I_{g, q}\left(A_{n}\right)+s\right) \geq 2\left(n g\left(a_{n}\right)-n N \log g\left(a_{n}\right)\right) \\
& >2\left(n g\left(a_{n}\right)-\frac{1}{4} n g\left(a_{n}\right)\right)=\frac{3}{2} n g\left(a_{n}\right) .
\end{aligned}
$$

Since $\frac{3}{2} n g\left(a_{n}\right) \geq \frac{3}{2} n a_{n}$ for large $n$, by (53) and since $x \rightarrow g(x)-$ $N \log g(x)$ is increasing, we have

$$
\begin{aligned}
g\left(x_{i}\right)-M\left(x_{i}\right) & \geq g\left(x_{i}\right)-N \log g\left(x_{i}\right) \geq g\left(2\left(I_{g, q}\left(A_{n}\right)+s\right)\right)-N \log g\left(2\left(I_{g, q}\left(A_{n}\right)+s\right)\right) \\
& >g\left(2\left(I_{g, q}\left(A_{n}\right)+s\right)\right)-\frac{1}{2} g\left(2\left(I_{g, q}\left(A_{n}\right)+s\right)\right) \\
& \geq \frac{1}{2}\left(2\left(I_{g, q}\left(A_{n}\right)+s\right)\right)=I_{g, q}\left(A_{n}\right)+s .
\end{aligned}
$$

Therefore since $\mathbf{x} \in F, x_{i} \leq 2\left(I_{g, q}\left(A_{n}\right)+s\right)$ for every $i$, which implicates that (66) holds. Thus we have

$$
S_{g, q}\left(I_{g, q}\left(A_{n}\right)+s\right) \subset H
$$

from which we deduce that

$$
\begin{aligned}
\text { Volume }\left(A_{n} \cap S_{g, q}\left(I_{g, q}\left(A_{n}\right)+s\right)\right) & \leq \operatorname{Volume}\left(S_{g, q}\left(I_{g, q}\left(A_{n}\right)+s\right)\right) \\
& \leq \operatorname{Volume}(H)=2^{n}\left(I_{g, q}\left(A_{n}\right)+s\right)^{n} .
\end{aligned}
$$

With this inequality, turning back to (49) it holds

$$
\begin{aligned}
\log P\left(A_{n}\right) & =\log c^{n} \int_{C} \exp \left(-\sum_{1 \leq i \leq n}\left(g\left(x_{i}\right)+q\left(x_{i}\right)\right)\right) d x_{1}, \ldots, d x_{n} \\
& =n \log c-I_{g, q}\left(A_{n}\right)+\log \int_{0}^{\infty} \operatorname{Volume}\left(A_{n} \cap S_{g, q}\left(I_{g, q}\left(A_{n}\right)+s\right)\right) e^{-s} d s \\
& \leq n \log c-I_{g, q}\left(A_{n}\right)+\log \int_{0}^{\infty}\left(I_{g, q}\left(A_{n}\right)+s\right)^{n} e^{-s} d s+n \log 2 .
\end{aligned}
$$


According to (43), it holds

$$
\int_{0}^{\infty}\left(I_{g, q}\left(A_{n}\right)+s\right)^{n} e^{-s} d s \leq(n+1) I_{g, q}\left(A_{n}\right)^{n},
$$

Hence we have

$$
\begin{aligned}
\log P\left(A_{n}\right) & \leq n \log c-I_{g, q}\left(A_{n}\right)+\log \left((n+1) I_{g, q}\left(A_{n}\right)^{n}\right)+n \log 2 \\
& =n \log c-I_{g, q}\left(A_{n}\right)+n \log I_{g, q}\left(A_{n}\right)+\log (n+1)+n \log 2 .
\end{aligned}
$$

which yields (65).

Step 4: In this step, we derive crude bounds for $I_{g_{2}}(C), I_{g_{1}}\left(I^{c} \cap C\right)$ and $I_{g_{2}}\left(I^{c} \cap C\right)$.

From (53) and (54), there exists some $a_{n} \in[X, \infty)$ (say, $\left.a_{n}>y_{2}\right)$ such that

$$
M(x) \leq \max \left(N \log g\left(a_{n}\right), N \log g(x)\right)
$$

holds on $(0, \infty)$. Hence for $a_{n}$ large enough

$$
g_{2}(x)=g(x)+M(x) \leq g(x)+\max \left(N \log g\left(a_{n}\right), N \log g(x)\right),
$$

which in turn yields

$$
I_{g_{2}}(C) \leq \inf _{\mathbf{x} \in C}\left(\sum_{i=1}^{n} g\left(x_{i}\right)+\sum_{i=1}^{n} \max \left(N \log g\left(a_{n}\right), N \log g\left(x_{i}\right)\right)\right) .
$$

It holds

$$
\inf _{\mathbf{x} \in C}\left(\sum_{i=1}^{n} \max \left(N \log g\left(a_{n}\right), N \log g\left(x_{i}\right)\right)\right)=n N \log g\left(a_{n}\right)
$$

which implies that

$$
\begin{aligned}
& \inf _{\mathbf{x} \in C}\left(\sum_{i=1}^{n} g\left(x_{i}\right)+\sum_{i=1}^{n} \max \left(N \log g\left(a_{n}\right), N \log g\left(x_{i}\right)\right)\right) \\
& =\inf _{\mathbf{x} \in C}\left(\sum_{i=1}^{n} g\left(x_{i}\right)\right)+\inf _{\mathbf{x} \in C}\left(\sum_{i=1}^{n} \max \left(N \log g\left(a_{n}\right), N \log g\left(x_{i}\right)\right)\right) \\
& =\inf _{\mathbf{x} \in C}\left(\sum_{i=1}^{n} g\left(x_{i}\right)\right)+n N \log g\left(a_{n}\right) \\
& =I_{g}(C)+n N \log g\left(a_{n}\right)=n g\left(a_{n}\right)+n N \log g\left(a_{n}\right) .
\end{aligned}
$$

Thus we obtain the inequality

$$
I_{g_{2}}(C) \leq n g\left(a_{n}\right)+n N \log g\left(a_{n}\right) .
$$


We now provide a lower bound of $I_{g_{1}}\left(I^{c} \cap C\right)$. Consider the inequality of (58) in Step 1, where we have showed that $h$ is convex for $x$ large enough; hence, using (58) when $a_{n}$ is sufficiently large, it holds

$$
I_{g_{1}}\left(I^{c} \cap C\right) \geq I_{h}\left(I^{c} \cap C\right)=\min \left(F_{h_{1}}\left(a_{n}, \epsilon_{n}\right), F_{h_{2}}\left(a_{n}, \epsilon_{n}\right)\right),
$$

where the second inequality holds from Lemma 1. By the definition of the function $h$ in (56), for large $x$ it holds $h(x)=r(x)$ which yields the following lower bound of $I_{g_{1}}\left(I^{c} \cap C\right)$

$$
I_{g_{1}}\left(I^{c} \cap C\right) \geq I_{h}\left(I^{c} \cap C\right)=I_{r}\left(I^{c} \cap C\right)=\min \left(F_{r_{1}}\left(a_{n}, \epsilon_{n}\right), F_{r_{2}}\left(a_{n}, \epsilon_{n}\right)\right) .
$$

By Lemma 1, it holds

$$
\begin{aligned}
& F_{r_{1}}\left(a_{n}, \epsilon_{n}\right)= g\left(a_{n}+\epsilon_{n}\right)+(n-1) g\left(a_{n}-\frac{1}{n-1} \epsilon_{n}\right) \\
&-N \log g\left(a_{n}+\epsilon_{n}\right)-(n-1) N \log g\left(a_{n}-\frac{1}{n-1} \epsilon_{n}\right) \\
& \geq g\left(a_{n}+\epsilon_{n}\right)+(n-1) g\left(a_{n}-\frac{1}{n-1} \epsilon_{n}\right)-n N \log g\left(a_{n}+\epsilon_{n}\right),
\end{aligned}
$$

by the same way, we have also

$$
F_{r_{2}}\left(a_{n}, \epsilon_{n}\right) \geq g\left(a_{n}-\epsilon_{n}\right)+(n-1) g\left(a_{n}+\frac{1}{n-1} \epsilon_{n}\right)-n N \log g\left(a_{n}+\epsilon_{n}\right),
$$

hence

$$
I_{g_{1}}\left(I^{c} \cap C\right) \geq \min \left(F_{g_{1}}\left(a_{n}, \epsilon_{n}\right), F_{g_{2}}\left(a_{n}, \epsilon_{n}\right)\right)-n N \log g\left(a_{n}+\epsilon_{n}\right)
$$

holds.

The method of the estimation of the upper bound of $I_{g_{2}}\left(I^{c} \cap C\right)$ is similar to that used for $I_{g_{2}}(C)$ above. In (68), replacing $C$ by $I^{c} \cap C$ we obtain

$$
\begin{aligned}
I_{g_{2}}\left(I^{c} \cap C\right) & \leq \inf _{\mathbf{x} \in I^{c} \cap C}\left(\sum_{i=1}^{n} g\left(x_{i}\right)+\sum_{i=1}^{n} \max \left(N \log g\left(a_{n}\right), N \log g\left(x_{i}\right)\right)\right) \\
& \leq \inf _{\mathbf{x} \in I^{c} \cap C}\left(\sum_{i=1}^{n} g\left(x_{i}\right)+\sum_{i=1}^{n} \max \left(N \log g\left(a_{n}+\frac{\epsilon_{n}}{n-1}\right), N \log g\left(x_{i}\right)\right)\right) .
\end{aligned}
$$


Similarly to (69), it holds

$\inf _{\mathbf{x} \in I^{c} \cap C}\left(\sum_{i=1}^{n} \max \left(N \log g\left(a_{n}+\frac{\epsilon_{n}}{n-1}\right), N \log g\left(x_{i}\right)\right)\right)=n N \log g\left(a_{n}+\frac{\epsilon_{n}}{n-1}\right)$,

where equality is attained setting $x_{1}=\ldots=x_{n-1}=a_{n}+\epsilon_{n} /(n-1), x_{n}=$ $a_{n}-\epsilon_{n}$. Hence we have, when $n \rightarrow \infty$

$$
\begin{aligned}
I_{g_{2}}\left(I^{c} \cap C\right) & \leq \inf _{\mathbf{x} \in I^{c} \cap C}\left(\sum_{i=1}^{n} g\left(x_{i}\right)+\sum_{i=1}^{n} \max \left(N \log g\left(a_{n}+\frac{\epsilon_{n}}{n-1}\right), N \log g\left(x_{i}\right)\right)\right) \\
& =\inf _{\mathbf{x} \in I^{c} \cap C} \sum_{i=1}^{n} g\left(x_{i}\right)+n N \log g\left(a_{n}+\frac{\epsilon_{n}}{n-1}\right) \\
& =I_{g}\left(I^{c} \cap C\right)+n N \log g\left(a_{n}+\frac{\epsilon_{n}}{n-1}\right) \\
& \leq g\left(a_{n}-\epsilon_{n}\right)+(n-1) g\left(a_{n}+\frac{1}{n-1} \epsilon_{n}\right)+n N \log g\left(a_{n}+\frac{\epsilon_{n}}{n-1}\right) \\
& \leq n g\left(a_{n}+\frac{\epsilon_{n}}{n-1}\right)+n N \log g\left(a_{n}+\frac{\epsilon_{n}}{n-1}\right) \\
& \leq n(N+1) g\left(a_{n}+\frac{\epsilon_{n}}{n-1}\right) .
\end{aligned}
$$

Therefore we obtain

$$
\log I_{g_{2}}\left(I^{c} \cap C\right) \leq \log n+\log (N+1)+\log g\left(a_{n}+\frac{\epsilon_{n}}{n-1}\right) .
$$

Step 5: In this step, we complete the proof by showing that

$$
\lim _{a_{n} \rightarrow \infty} \frac{P\left(I^{c} \cap C\right)}{P(C)}=0 .
$$

Using the upper bound of $P\left(I^{c} \cap C\right)$, together with the lower bound of $P(C)$ above, we have when $a_{n}$ is large enough

$$
\begin{aligned}
\frac{P\left(I^{c} \cap C\right)}{P(C)} & \leq \exp \left(\begin{array}{c}
-\left(I_{g, q}\left(I^{c} \cap C\right)-I_{g, q}(C)\right)+n \log I_{g, q}\left(I^{c} \cap C\right) \\
+\tau_{n}+n \log g\left(a_{n}\right)+\log (n+1)+n \log 2
\end{array}\right) \\
& \leq \exp \left(-\left(I_{g, q}\left(I^{c} \cap C\right)-I_{g, q}(C)\right)+n \log I_{g, q}\left(I^{c} \cap C\right)+\tau_{n}+2 n \log g\left(a_{n}\right)\right) \\
& \leq \exp \left(-\left(I_{g_{1}}\left(I^{c} \cap C\right)-I_{g_{2}}(C)\right)+n \log I_{g_{2}}\left(I^{c} \cap C\right)+\tau_{n}+2 n \log g\left(a_{n}\right)\right) .
\end{aligned}
$$

The last inequality holds from (51) and (52). Replace $I_{g_{1}}\left(I^{c} \cap C\right), I_{g_{2}}(C)$ by the upper bound of (70) and the lower bound of (71), respectively, 
we obtain

$$
\begin{aligned}
I_{g_{1}}\left(I^{c} \cap C\right)-I_{g_{2}}(C) \geq \min \left(F_{g_{1}}\left(a_{n}, \epsilon_{n}\right), F_{g_{2}}\left(a_{n}, \epsilon_{n}\right)\right)-n N \log g\left(a_{n}+\epsilon_{n}\right) \\
\quad-\left(n g\left(a_{n}\right)+n N \log g\left(a_{n}\right)\right) \\
=H\left(a_{n}, \epsilon_{n}\right)-n N \log g\left(a_{n}+\epsilon_{n}\right)-n N \log g\left(a_{n}\right) \\
\geq H\left(a_{n}, \epsilon_{n}\right)-2 n N \log g\left(a_{n}+\epsilon_{n}\right) .
\end{aligned}
$$

Under condition (7), there exists some $Q$ such that $n \log n \leq Q n \log g\left(a_{n}\right)$, which, together with (72) and (74), gives

$$
\begin{aligned}
\frac{P\left(I^{c} \cap C\right)}{P(C)} & \leq \exp \left(\begin{array}{c}
-H\left(a_{n}, \epsilon_{n}\right)+n(2 N+1) \log g\left(a_{n}+\epsilon_{n}\right) \\
+\tau_{n}+2 n \log g\left(a_{n}\right)+n \log n+n \log (N+1)
\end{array}\right) \\
& \leq \exp \left(-H\left(a_{n}, \epsilon_{n}\right)+n(2 N+2 Q+3) \log g\left(a_{n}+\epsilon_{n}\right)+\tau_{n}\right) .
\end{aligned}
$$

The second term in the exponent in the last line above and $\tau_{n}$ are both of small order with respect to $H\left(a_{n}, \epsilon_{n}\right)$. Indeed under condition (11), when $a_{n} \rightarrow \infty$, it holds

$$
\lim _{n \rightarrow \infty} \frac{n(2 N+2 Q+3) \log g\left(a_{n}+\epsilon_{n}\right)}{H\left(a_{n}, \epsilon_{n}\right)}=0 .
$$

For $\tau_{n}$ which is defined in (63) under conditions (11), (12), $n N \log g\left(a_{n}\right)$ and $n G\left(a_{n}\right)$ are both of smaller order than $H\left(a_{n}, \epsilon_{n}\right)$. As regards to the third term of $\tau_{n}$, it holds

$$
\begin{aligned}
n N \log g\left(a_{n}+\frac{1}{g\left(a_{n}\right)}\right) & =n N \log \left(g\left(a_{n}+\frac{1}{g\left(a_{n}\right)}\right)-g\left(a_{n}\right)+g\left(a_{n}\right)\right) \\
& \leq n N \log \left(2 \max \left(G\left(a_{n}\right), g\left(a_{n}\right)\right)\right) \\
& =n N \log 2+\max \left(n N \log G\left(a_{n}\right), n N \log g\left(a_{n}\right)\right) .
\end{aligned}
$$

Under conditions (11) and (12), both $n N \log G\left(a_{n}\right)$ and $n N \log g\left(a_{n}\right)$ are small with respect to $H\left(a_{n}, \epsilon_{n}\right)$; therefore $n N \log g\left(a_{n}+1 / g\left(a_{n}\right)\right)$ is small with respect to $H\left(a_{n}, \epsilon_{n}\right)$ when $a_{n} \rightarrow \infty$. Hence it holds when $a_{n} \rightarrow \infty$

$$
\lim _{n \rightarrow \infty} \frac{\tau_{n}}{H\left(a_{n}, \epsilon_{n}\right)}=0 .
$$

Finally, (75), together with (76) and (77), implies that (73) holds.

\section{References}

[1] Barbe, Ph., Broniatowski, M.: Blowing number of a distribution for a statistics and loyal estimators. Statist. Probab. Lett. 69 , no. 4, 465-475 (2004) 
[2] Bingham, N. H.; Goldie, C. M.; Teugels, J. L. :Regular variation. Encyclopedia of Mathematics and its Applications, 27. Cambridge University Press, Cambridge, xx+494 pp., (1989)

[3] Broniatowski, M., Fuchs, A. : Tauberian theorems, Chernoff inequality, and the tail behavior of finite convolutions of distribution functions. Adv. Math. 116, no. 1, 12-33, (1995)

[4] Broniatowski, M., Cao, Z. : A conditional limit theorem for random walks under extreme deviation. arXiv:1206.6951 (2012)

[5] Cao, Z. : An Abelian theorem with application to the conditional Gibbs principle. arXiv:1302.1337 (2013)

[6] de Haan, L., Ferreira, A., Extreme value theory. An introduction. Springer Series in Operations Research and Financial Engineering. Springer, New York, xviii+417 pp., (2006)

[7] Feigin, P. D.; Yashchin, E. : On a strong Tauberian result. Z. Wahrsch. Verw. Gebiete 65, no. 1, 35-48, (1983)

[8] Frisch, U., Sornette, D.: Extreme deviations and Applications. J. Phys.I, France, 7 (1997)

[9] Sornette, D.: Critical phenomena in natural sciences, Springer series in Synergetics, 2d Edition (2006)

[10] Jensen, J.L. : Saddlepoint Approximations. Oxford Statistical Science Series, vol 16. The Clarendon Press Oxford University Press, New York, Oxford Science Publications (1995)

[11] Juszczak, D. , Nagaev, A. : Local large deviation theorem for sums of I.I.D. random vectors when the Cramér condition holds in the whole space. Probab. Math. Statist. 24, no. 2, Acta Univ. Wratislav. No. 2732, 297-320, (2004)

[12] Mason, David M. An extended version of the Erdős-Rényi strong law of large numbers. Ann. Probab. 17 , no. 1, 257-265, (1989) 